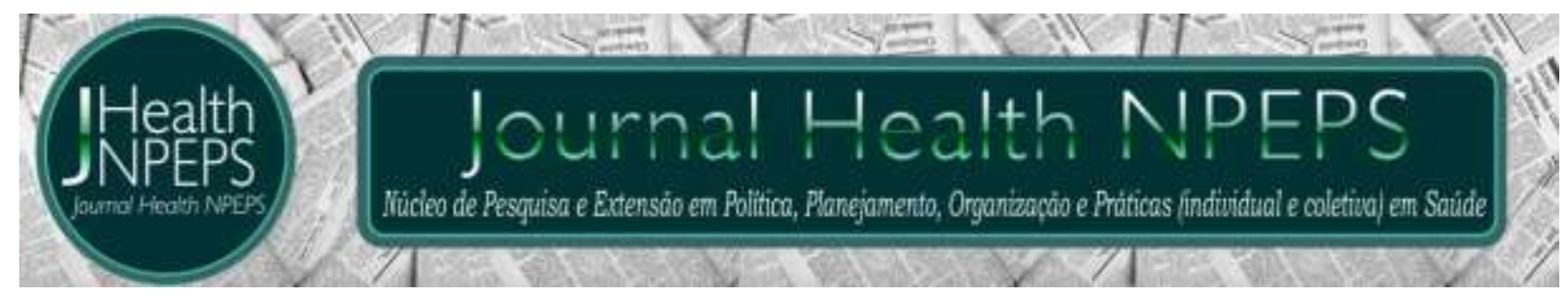

http://dx.doi.org/10.30681/252610103444

ARTIGO ORIGINAL

\title{
Conhecimento de pediatras e residentes sobre fatores de risco associados ao glaucoma cortisônico
}

\section{Knowledge of pediatricians and residents about risk factors associated with cortisone glaucoma}

\section{Conocimiento de pediatras y residentes sobre factores de riesgo asociados al glaucoma cortisónico}

\section{Raquel Bragança Soares Capacia ${ }^{1}$, Renato Ribeiro Nogueira Ferraz ${ }^{2}$}

\section{RESUMO}

Objetivo: avaliar o conhecimento de pediatras e residentes sobre fatores de risco associados ao glaucoma cortisônico. Método: trata-se de um estudo descritivo, quantitativo e prospectivo, realizado em novembro de $2018 \mathrm{com}$ médicos pediatras e residentes de pediatria atuantes na cidade Rio de Janeiro, Brasil, que responderam a um questionário com 12 questões sobre o seu nível de conhecimento em relação aos fatores de risco para o glaucoma cortisônico. As variáveis foram apresentadas por frequência absoluta e relativa, e por medida de tendência central seguida de uma medida de dispersão. Resultados: a maioria dos participantes desconhecem a relação entre o uso de corticosteroides e o desenvolvimento de glaucoma secundário, desconhecem os fatores de risco associados ao desenvolvimento da referida doença, e não encaminham os pacientes sob uso de corticoides para acompanhamento com o oftalmologista. Conclusão: verifica-se a necessidade de criação de campanhas visando informar os médicos sobre a importância de se prescrever os corticosteroides com cautela, e quando a prescrição for necessária. Ressalta-se também, a importância da avaliação por profissional habilitado, especialmente buscando evitar o desenvolvimento do glaucoma cortisônico.

Descritores: Pediatria; Oftalmologia; Glaucoma; Corticosteroides.

\footnotetext{
${ }^{1}$ Médica. Especialista em Oftalmologia. Médica do Setor de Estrabismo e Oftalmologia Pediatrica - Hospital Municipal da Piedade, Rio de Janeiro, RJ, Brasil. E-mail: raquelbraganca@yahoo.com.br ORCID ID: https://orcid.org/0000-0002-0642-5531 Autor principal - Endereço para correspondência: R. da Capella, 96 Piedade, Rio de Janeiro - RJ, 20740-310.

${ }^{2}$ Biólogo. Doutor em Ciências. Professor Permanente do Mestrado Profissional em Administração - Gestão em Sistemas de Saúde (PMPA-GSS) - Universidade Nove de Julho (UNINOVE). São Paulo, SP, Brasil. E-mail: renatobio@hotmail.com ORCID ID: https://orcid.org/0000-0002-8931-895X
} 


\section{ABSTRACT}

Objective: to evaluate the pediatricians and pediatric residents' knowledge regarding the risk factors associated with cortisone-induced glaucoma. Method: this is a descriptive, quantitative and prospective study conducted in November 2018 with pediatricians and pediatric residents in the city of Rio de Janeiro, Brazil, who answered a questionnaire with 12 questions about their level of knowledge in relation to the risk factors for cortisone glaucoma. The variables were presented by absolute and relative frequency, and by central tendency measure followed by a dispersion measure. Results: most respondents are unaware of the relationship between the use of corticosteroids and the development of secondary glaucoma, do not know the risk factors associated with the development of this disease, and do not refer patients using corticosteroids for a follow-up with the ophthalmologist. Conclusion: it is necessary to create campaigns to inform physicians about the importance of caution in the corticosteroids prescription, especially in order to avoid the development of cortisone-induced glaucoma.

Descriptors: Pediatrics; Ophthalmology; Glaucoma; Corticosteroids.

\section{RESUMEN}

Objetivo: evaluar el conocimiento de pediatras y residentes sobre factores de riesgo asociados al glaucoma cortisónico. Método: se trata de un estudio descriptivo, cuantitativo y prospectivo, realizado en noviembre de 2018 con médicos pediatras y residentes de pediatría actuantes en la ciudad Río de Janeiro, Brasil, que respondieron a un cuestionario con 12 preguntas sobre el nivel de conocimiento de los participantes en relación a los factores de riesgo para el glaucoma cortisónico. Las variables fueron presentadas por frecuencia absoluta y relativa, y por medida de tendencia central seguida de una medida de dispersión. Resultados: la mayoría de los participantes desconocen la relación entre el uso de corticosteroides y el desarrollo de glaucoma secundario, desconocen los factores de riesgo asociados al desarrollo de la referida enfermedad, y no encaminan a los pacientes sobre el uso de corticoides para acompañamiento con el oftalmólogo. Conclusión: se verificó la necesidad de crear campañas para informar a los médicos sobre la importancia de prescribir los corticosteroides con cautela, y cuando la prescripción sea necesaria. Se resalta también la importancia de la evaluación por profesional habilitado, especialmente buscando evitar el desarrollo del glaucoma cortisónico.

Descriptores: Pediatría; Oftalmología; Glaucoma; Corticosteroides.

INTRODUÇÃO

O glaucoma cortisônico é um tipo de glaucoma secundário induzido pelo uso de esteroides locais ou orais, normalmente prescrito para tratamento de doenças inflamatórias crônicas ${ }^{1}$. 0 uso indiscriminado de corticosteroides, especialmente em formulações de colírio, tem se tornado um hábito bastante comum, especialmente pelo fato de poder ser adquirido em drogarias sem a necessidade de prescrição médica, 
gerando uma série de efeitos indesejados ${ }^{2}$. Dentre os efeitos colaterais oculares, catarata e glaucoma são os mais comuns². Hipertensão ocular induzida por esteroides foi relatada já na década de 1950, quando o uso prolongado de esteroides sistêmicos mostrou a capacidade de aumentar a pressão intraocular ${ }^{3}$.

Em um estudo realizado em um hospital terciário do norte da Índia (IN), com amostra aleatória de 119 funcionários, incluindo 23 médicos (não-oftalmologistas) e $\quad 96$ trabalhadores de enfermagem, verificou-se que o conhecimento sobre - glaucoma estava abaixo do esperado 4 .

Em outro estudo realizado em Minas Gerais (BR) com 102 acadêmicos do $5^{\circ}$ e $6^{\circ}$ ano de um curso de medicina, identificou-se que a maioria dos participantes acreditam que os conhecimentos fornecidos na graduação sobre o glaucoma são efetivamente suficientes ${ }^{5}$.

No Brasil, ainda não há nenhum estudo que buscou conhecer particularidades sobre o glaucoma entre médicos, especialmente sobre formas secundárias da doença. Assim, este trabalho pretende responder à seguinte questão de pesquisa: qual é o nível de conhecimento dos pediatras e residentes em pediatria com relação aos fatores de risco associados ao glaucoma cortisônico? Para isso, o objetivou-se avaliar o conhecimento de pediatras e residentes sobre fatores de risco associados ao glaucoma cortisônico.

\section{MÉTODO}

Trata-se de uma pesquisa descritiva e com abordagem quantitativa, realizada com médicos pediatras e residentes de pediatria atuantes na cidade do Rio de Janeiro RJ, Brasil, no período de novembro de 2018.

0 instrumento de coleta de dados resumiu-se a um questionário contendo 12 perguntas, sendo 11 delas fechadas e uma aberta. As questões buscaram identificar o nível de conhecimento dos participantes em relação aos fatores de risco para o desenvolvimento do glaucoma cortisônico, além de traçar o perfil dos voluntários em relação ao sexo, idade e tempo de exercício da medicina.

Os participantes, que constituíram uma amostra de 
conveniência, faziam parte dos contatos pessoais de um dos autores da presente pesquisa, que possuía o número telefônico de alguns e $\mathrm{o} e$ mail pessoal de outros, sendo estes convidados a participar por meio do contato por uma das duas formas citadas. O único critério de inclusão referiu-se ao fato de que os participantes obrigatoriamente precisariam possuir o título de médico pediatra, ou serem residentes em pediatria. Nenhuma Universidade ou Instituição de Saúde foi envolvida no estudo.

Os participantes do estudo, integraram a amostra por meio de acesso eletrônico a um formulário construído no Google Forms, contendo em sua primeira tela o Termo de Consentimento Livre e Esclarecido (TCLE) para leitura e opção de aceite em iniciar 0 estudo. Nenhuma informação que pudesse identificar os participantes individualmente foi coletada e as respostas foram analisadas apenas em conjunto.

O tempo de preenchimento do questionário foi de aproximadamente cinco minutos entre os participantes. Nenhum participante que se prontificou a participar do estudo desistiu, não havendo nenhuma exclusão.

Inicialmente as variáveis avaliadas foram apresentadas apenas de forma descritiva, e um teste simples de correlação foi aplicado para verificar possíveis relações entre as variáveis coletadas. Os cálculos foram realizados no programa SPSS versão 22.0. As variáveis categóricas foram apresentadas por frequência absoluta seguida de sua respectiva frequência relativa para cada uma das questões. Variáveis numéricas foram apresentadas por medida de tendência central seguida de sua respectiva medida de dispersão, dependendo da forma de distribuição dos dados, se paramétrica ou não paramétrica.

Este trabalho foi submetido ao Comitê de Ética em Pesquisa em Pesquisa com Seres Humanos (CEP) da Universidade Nove de Julho, instituição de origem de um dos autores deste trabalho, seguindo as diretrizes previstas na resolução 466/2012 do Conselho Nacional de Saúde (CNS), que define os aspectos éticos e legais das pesquisas envolvendo seres humanos. 0 estudo teve como registro o CAAE $n$. 04095518.0.0000.5511 e com parecer 
de aprovação n. 3.139.486. Todos participantes do estudo assinaram 0 TCLE.

\section{RESULTADOS}

A amostra deste estudo foi constituída por 56 participantes. A média de idade foi de $36,5 \pm 12,5$ anos, com mediana de 36,5 anos (mínimo de 24 e máximo de 67 anos). Quanto ao tempo de exercício da medicina, observou-se uma média de $11 \pm 12$ anos, com mediana de 11 anos (mínimo de 1 e máximo de 44 anos). Dos participantes, 52 (93\%) eram mulheres. Em relação a categoria, 47 $(84 \%)$ eram pediatrias, $2(3,5 \%)$ eram R1 em pediatria e $7(12,5 \%)$ eram R2 em pediatria.

Todos os participantes alegaram prescrever corticosteroides no exercício diário da profissão. Quanto às vias de administração dos medicamentos, destacou a via oral, seguida da injetável.

$\mathrm{Na}$ avaliação da frequência das prescrições, a maioria dos participantes alegou prescrever os corticosteroides mais de uma vez por semana, seguida da frequência de uma vez por mês, uma vez por semana, e mais de uma vez por mês.
Quando avaliada a duração da prescrição, ou seja, o período de uso, a maioria dos participantes alegou prescrever os medicamentos pelo período de sete dias, intervalo este seguido das prescrições por 10 dias, depois pelas prescrições contínuas, pelas prescrições de uso uma vez por semana, e por aquelas maiores ou iguais a 14 dias.

Figura 1 - Vias de administração das prescrições. Novembro de 2018. Rio de Janeiro-RJ, Brasil.

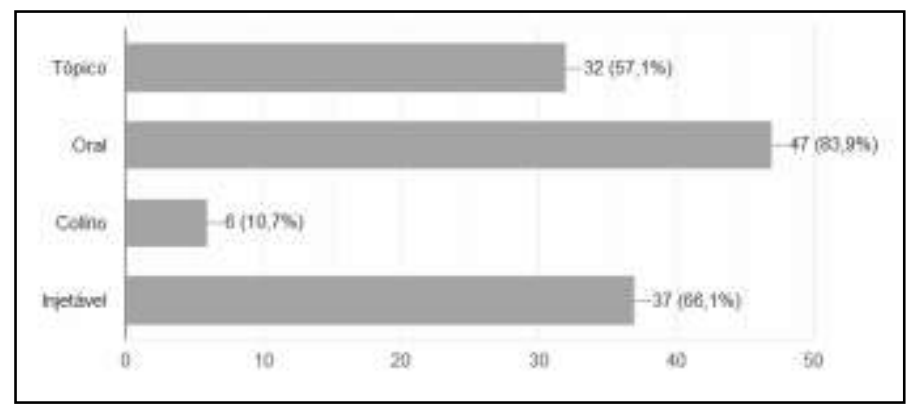

Figura 2 - Frequência de prescrição dos corticosteroides. Novembro de 2018. Rio de JaneiroRJ, Brasil.

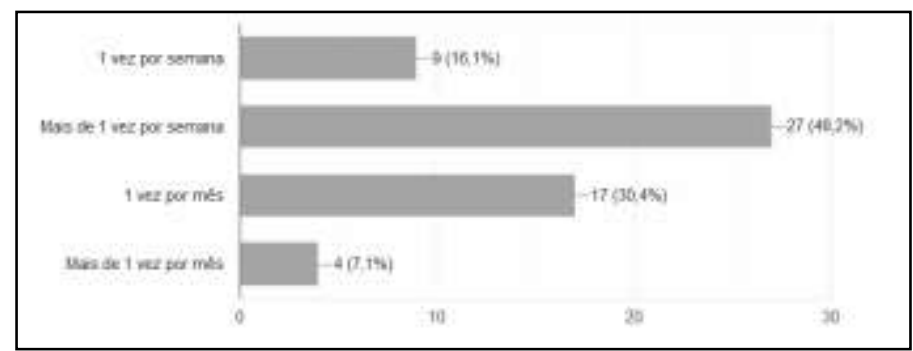

Figura 3 - Duração da prescrição de corticosteroides (período de uso). Novembro de 2018. Rio de JaneiroRJ, Brasil.

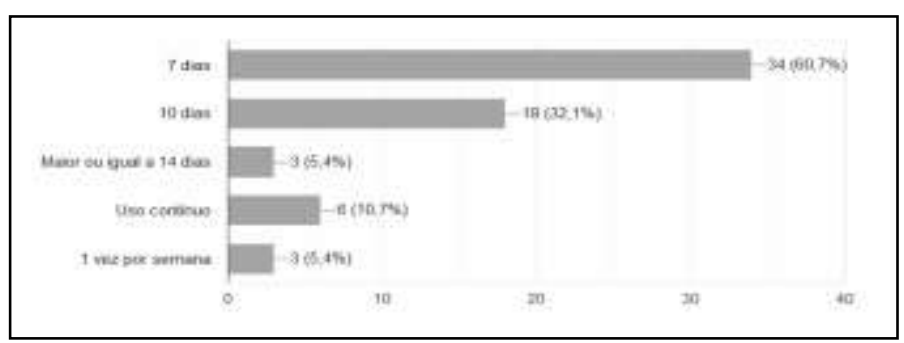


$\mathrm{Na}$ avaliação da questão norteadora, que se referia ao fato do participante conhecer ou não o glaucoma cortisônico, apenas 20 (35\%) participantes responderam conhecer a doença.

Quando indagados se os participantes, ao prescreverem a corticoterapia, encaminham o paciente para acompanhamento do médico oftalmologista, a maioria dos participantes respondeu nunca realizar o encaminhamento, seguidos daqueles que sempre encaminham ou que só encaminham quando a prescrição é de uso contínuo, e por aquele que realizam o encaminhamento apenas se o tempo de uso for superior a 10 dias.

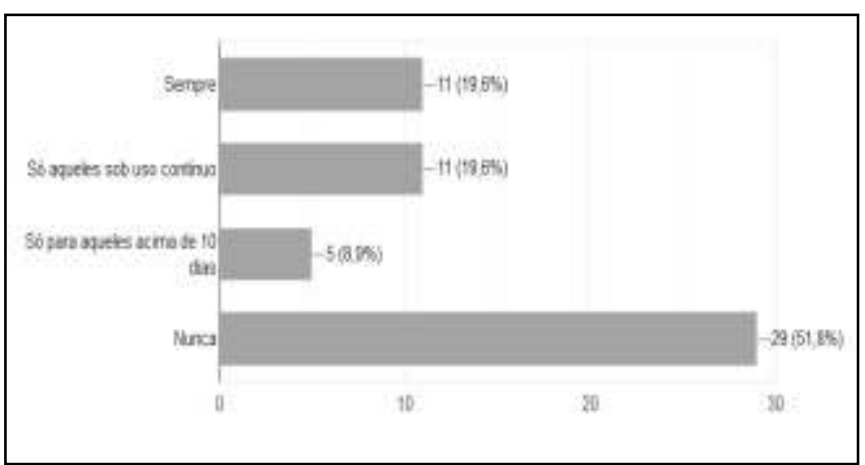

Figura 4 - Frequência de encaminhamento ao oftalmologista. Novembro de 2018. Rio de Janeiro-RJ, Brasil.

A avaliação das respostas em relação ao conhecimento dos fatores de risco associados ao glaucoma cortisônico, 48 (85,7\%) participantes disseram não os conhecer, enquanto apenas $8(14,3 \%)$ disseram conhecer esses fatores de risco.

\section{DISCUSSÃO}

O glaucoma decorrente do uso de corticosteroides é conhecido no meio médico como glaucoma cortisônico ${ }^{1}$. Dentre os diversos efeitos colaterais do uso destes medicamentos, ressaltam-se os defeitos oculares, especialmente a catarata e o próprio glaucoma ${ }^{2}$. Estas doenças ocorrem principalmente devido ao aumento da pressão intraocular, comum na corticoterapia 3 .

No presente estudo, a grande maioria dos participantes, correspondendo a mais de dois terços da amostra, desconheciam a existência do glaucoma induzido pelo uso de corticosteroides. Um estudo que buscou identificar a frequência de catarata infantil em um Serviço de oftalmologia localizado no Estado de Minas Gerais, após a avaliação dos 44 casos, identificou frequência de glaucoma secundário em quase $20 \%$. A despeito do fato, de que a conclusão dos autores tenha girado especialmente em torno da catarata 
congênita, verificou-se elevado percentual de crianças com glaucoma, cuja etiologia não foi identificada pelos idealizadores do estudo. Talvez, a avaliação do uso prolongado ou em doses elevadas de corticosteroides possa ter se associado, à época, com a elevada taxa de glaucoma secundário6.

Mais da metade dos participantes do estudo não encaminha os pacientes sob uso de corticosteroides para avaliação com oftalmologista. Em 2018, em um estudo conduzido em Salvador (BA), buscou-se descrever o perfil clínico e epidemiológico de pacientes com hipertensão intraocular após a realização de cirurgia para colocação de válvula de Ahmed. Quase metade dos 40 pacientes acompanhados apresentaram o glaucoma secundário como doença de base ${ }^{7}$.

Em ambos os estudos ${ }^{6,7}$, os autores não indagaram os pacientes sobre o uso de corticoides, ou mesmo se estes haviam sido encaminhados anteriormente ao acompanhamento de oftalmologistas, ressaltando a necessidade de disseminar tal informação junto à categoria médica visando prevenir a possível ocorrência de glaucoma secundário.
A grande maioria dos participantes desconhece os fatores de risco que podem estar associados ao desenvolvimento de glaucoma cortisônico. Sabe-se que o glaucoma corresponde a um dos principais fatores para ocorrência de cegueira no Brasil, com tendência a um aumento significativo da sua incidência no futuro, principalmente devido ao envelhecimento natural da população. Este fato, além de trazer uma série de prejuízos ao paciente e à sua família, também tende a onerar consideravelmente os sistemas de saúde, tornando de suma importância a sua prevenção ${ }^{8}$. Em somatória, é fato que o glaucoma gera medo e preocupação no paciente relacionado tanto à possibilidade de desenvolvimento de cegueira, quanto à dificuldade de tratamento da doença, afetando consideravelmente a sua qualidade de vida 9 .

Dos poucos participantes que se arriscaram em fornecer alguns fatores de risco para o desenvolvimento da doença, o uso prolongado, uso frequente e as altas doses foram adequadamente mencionados. Todavia, o fato mais marcante do estudo foi a elevada taxa de profissionais que desconhecem tais 
fatores de risco. Vale lembrar que pacientes com glaucoma induzido por corticoides e com idade menor do que 20 anos apresentam elevada chance de necessidade de correção cirúrgica ${ }^{10}$.

Não é novidade que a utilização de medicamentos por conta própria pode trazer sérios prejuízos a saúde de seus usuários. Por exemplo, estudo recente realizado em Feira de Santana (BA), identificou-se que mais de $50 \%$ dos participantes faziam uso de corticosteroides sem prescrição médica ${ }^{11}$.

Dessa forma, além de programas de orientação para os médicos, é necessário alertar também os farmacêuticos e a própria população quanto aos possíveis efeitos colaterais do uso de corticosteroides, com destaque para o glaucoma cortisônico.

\section{CONCLUSÃO}

A maioria dos participantes desconhece a relação entre o uso de corticosteroides e o desenvolvimento de glaucoma secundário, desconhece os fatores de risco associados ao desenvolvimento da referida doença, e não encaminha os pacientes sob uso de corticoides para acompanhamento com o oftalmologista.

Os resultados do presente trabalho chamam a atenção para a necessidade de criação de campanhas visando informar os médicos sobre a importância de se prescrever os corticosteroides com cautela, e somente quando a prescrição for efetivamente necessária. Ressalta-se também a importância da avaliação por profissional habilitado, visto que além dos diversos efeitos colaterais já conhecidos e que podem ser oriundos da utilização destes medicamentos, existe também a possibilidade de instalação do glaucoma cortisônico.

Dentre as principais limitações do presente estudo podem ser citados o fato de ter sido realizado apenas pontualmente em um único Centro, e ainda com a utilização de uma amostra de conveniência. Nesse sentido, sugere-se a realização de novos estudos controlados e com amostra previamente calculada visando verificar se os resultados aqui observados poderão se repetir quando avaliados em maior escala.

\section{REFERÊNCIAS}


1. El MA, Mazlout $\mathrm{H}$, Trojet $\mathrm{S}$, Larguech L, Megaieth K, Belhaj S, et al. Cortisone glaucoma: epidemiological, clinical, and therapeutic study. J Fr Ophtalmol. 2007; 30(1):49-52.

2. Phulke S, Kaushik S, Kaur S, Pandav S. Steroid-induced Glaucoma: An Avoidable Irreversible Blindness. J Curr Glaucoma Pract. 2017; 11(2):6772.

3. McLean JM. Clinical and experimental observation on the use of $\mathrm{ACTH}$ and cortisone in ocular inflammatory disease. Trans Am Ophthalmol. 1950; 48:259-296.

4. Ichhpujani P, Bhartiya S, Kataria M, Topiwala P. Knowledge, Attitudes and Self-care Practices associated with Glaucoma among Hospital Personnel in a Tertiary Care Center in North India. J Curr Glaucoma Pract. 2012; 6(3):10812.

5. Martins SC, Mendes MH, Guedes RA, Guedes VM, Chaoubah A. Nível de conhecimento sobre glaucoma primário de ângulo aberto entre os estudantes de Medicina. Rev Bras Oftalmol. 2014; 73(5):302-7.
6. Mérula RV, Fernandes LC. Catarata infantil: importância do diagnóstico e tratamento precoces. Arq Bras Oftalmol. 2005; 68(3):299-305.

7. Vilas-Boas FS, Fernandes MB, Ladeia JS. Perfil clínicoepidemiológico de pacientes com pico hipertensivo após implante de válvula de Ahmed. Rev Bras Oftalmol. 2018; 77(5):235-9.

8. Guedes RAP, Guedes VMP, Chaoubah A. Custo-efetividade no glaucoma. Conceitos, resultados e perspectiva atual. Rev Bras Oftalmol. 2018; 75(4):336-341.

9. Guedes RAP, Guedes RAP. Quality of life and glaucoma. Rev Bras Oftalmol. 2015; 74(3):131-2.

10. Sihota R, Konkal VL, Dada T, Agarwal HC, Singh R. Prospective, long-term evaluation of steroidinduced glaucoma. Eye (lond). 2008; 22(1):26-30.

11. Almeida $A A B$, Andrade CRB. Uso indiscriminado dos corticosteróides no manejo das doenças respiratórias em uma drogaria em Feira de Santana BA: ênfase na rinite alérgica. Textura. 2018; 11(20):056-064. 
Conflito de interesses: Os autores declaram não haver conflito de interesses.

\section{Participação dos autores:}

- Concepção: Capacia RBS, Ferraz RRN.

- Desenvolvimento: Capacia RBS, Ferraz RRN.

- Redação e revisão: Capacia RBS, Ferraz RRN.

Como citar este artigo: Capacia RBS, Ferraz RRN. Conhecimento de pediatras e residentes sobre fatores de risco associados ao glaucoma cortisônico. J Health NPEPS. 2019; 4(1):113122.

Submissão: 03/02/2019

Aceito: $25 / 05 / 2019$

Publicado: 01/06/2019 